\title{
Editorial for the Special Issue on Photometric Analysis for Computer Vision
}

\author{
Peter Belhumeur • Katsushi Ikeuchi • \\ Emmanuel Prados • Stefano Soatto • Peter Sturm
}

Received: 14 May 2009 / Accepted: 25 August 2009 / Published online: 11 September 2009

(C) Springer Science+Business Media, LLC 2009

This special issue arises from papers presented at the Workshop on Photometric Analysis For Computer Vision, held on October 14, 2007 in conjunction with the 11th International Conference on Computer Vision Conference in Rio de Janeiro, Brazil. The workshop featured 7 oral presentations, 10 posters, and a keynote lecture by Todd Zickler. Papers submitted to this special issue underwent the full review cycle of IJCV.

Photometric analysis is a central aspect of computer vision theory and practice. The way an image looks depends on many factors, including geometry, illumination and reflectance properties of the objects. For transparent or translucent objects, or for objects composed by multiple coatings, the factors are even more numerous (refraction, subsurface scattering, ...). The laws combining these components are diverse and complex. This complexity makes computer vision tasks even more difficult and typically causes the failure of methods based on simple models.

A typical example could be problems caused by specularities in the stereo-vision problem; proposed methods usually assume that the scene is perfectly diffuse. Feature tracking and matching is another example since the photometric

P. Belhumeur

Columbia University, New York, NY 10027, USA

K. Ikeuchi

University of Tokyo, 4-6-1 Komaba, Meguro-ku, Tokyo, Japan

E. Prados $(\varangle) \cdot$ P. Sturm

INRIA Grenoble-Rhône-Alpes, 655 Avenue de l'Europe,

Montbonnot, 38334 Saint Ismier Cedex, France

e-mail: Emmanuel.Prados@inrialpes.fr

S. Soatto

UCLA, 405 Hilgard ave, Los Angeles, USA appearance of objects can change when they or the camera move.

On the one hand, from a theoretical as well as from a computational point of view, a better understanding and handling of these factors should improve robustness to photometric effects. On the other hand, this allows not only to circumvent problems but also to gather valuable information which can be practically exploited in computer vision tasks. One example is the information provided by shading and shadows.

This special issue contains 9 papers that cover several important aspects of photometric analysis, ranging from color analysis, shading, illumination, material reflectance, to perceived transparency due to motion blur and specialized appearance models for weather conditions such as rain and snow. The paper by Arjan Gijsenij, Theo Gevers, and Joost van de Weijer considers the problem of color constancy; a novel extension of the gamut mapping approach is presented which incorporates the statistical nature of (derivative) images. Four papers are dedicated to estimating material reflectance properties of objects from images. An active approach is proposed by Chi Cui, Hyunjin Yoo, and Moshe Ben-Ezra. This approach obtains multispectral reflectance properties in relatively uncontrolled settings and without special equipment, using an optimized wide band illumination. William A.P. Smith and Edwin R. Hancock show how to estimate the shape and reflectance properties of a face from a single image. Daisuke Miyazaki, Takushi Shibata, and Katsushi Ikeuchi propose a method to record the appearance of the objects for photorealistic rendering, based on polarization, parametric reflection model, and Daubechies Wavelet. Kuk-Jin Yoon, Emmanuel Prados, and Peter Sturm present a method for the joint estimation of object shape and reflectance from multiple images with known illumination 
conditions that naturally combines stereo and shading information. Shading is also used in the work by Chenyu Wu, Srinivasa G. Narasimhan, and Branislav Jaramaz which, introduce a multi-view shape-from-shading approach dedicated to perspective endoscopes, for the reconstruction of the surface of bones. Daisuke Miyazaki, Kenji Hara, and Katsushi Ikeuchi show how to apply Photometric Stereo to specular objects seen through glass. In the paper by Vincenzo Caglioti and Alessandro Giusti, the authors show how to model and analyze motion blur and the induced apparent transparency using alpha mattes. Peter C. Barnum, Srinivasa G. Narasimhan, and Takeo Kanade show how to remove weather effects from images due to rain and snow, using a dedicated appearance model combined with a statistical analysis in frequency space.

We believe that this special issue consists of a set of exciting papers, demonstrating the benefits of using advanced models and methods in the photometric analysis underlying various computer vision tasks. We thank the authors for their contributions and all the reviewers for their effort and time. 